\title{
BMJ Open Impact of surgical management of primary tumors in stage IV breast cancer patients: a retrospective observational study based on SEER database
}

\author{
Ning Xie, ${ }^{1}$ Xiaobo Hu, ${ }^{2}$ Yu Tang (D) , ${ }^{1}$ Can Tian, ${ }^{1}$ Ying He, ${ }^{2}$ Zhe-Yu Hu (D) , ${ }^{1}$ \\ Chongyu Hu, ${ }^{3}$ Xiao Wang, ${ }^{4}$ Xiangyan Liu, ${ }^{5,6}$ Liping Liu, ${ }^{1}$ Huawu Xiao, ${ }^{1}$ \\ Wei Peng (D) , ${ }^{2}$ Haoyu Zhou, ${ }^{7}$ Quchang Ouyang ${ }^{1}$
}

To cite: Xie N, Hu X, Tang Y, et al. Impact of surgical management of primary tumors in stage IV breast cancer patients: a retrospective observational study based on SEER database. BMJ Open 2022;12:e054135. doi:10.1136/ bmjopen-2021-054135

- Prepublication history and additional supplemental material for this paper are available online. To view these files, please visit the journal online (http://dx.doi.org/10.1136/ bmjopen-2021-054135)

Mr. Yu Tang left.

Received 08 June 2021 Accepted 15 December 2021

Check for updates

(c) Author(s) (or their employer(s)) 2022. Re-use permitted under CC BY-NC. No commercial re-use. See rights and permissions. Published by BMJ.

For numbered affiliations see end of article.

Correspondence to Dr Wei Peng; pengwei19810404@163.com, Dr Haoyu Zhou; zhy@hunau.edu.cn and Quchang Ouyang; oyqc1969@126.com

\section{ABSTRACT}

Objectives Although primary tumour surgery could prolong survival for patients with stage IV breast cancer, how to select candidates for primary tumour surgery is still a challenging problem for medical oncologists.

Design This study is a retrospective database study. Setting and participants In this study, we aimed at evaluating the primary site surgery effect and select the beneficial subgroups. 13618 patients with stage IV breast cancer, diagnosed between 2010 and 2015, were collected from SEER*Stat database.

Interventions Based on the local surgery at primary tumour site, patients were categorised into three groups: primary tumour surgery performed group, recommended for primary tumour surgery but refused (RBR) group and surgery not recommended (NR) group.

Primary and secondary outcome measures All-cause survival and breast cancer-specific survival (BCSS). Results Univariate Cox regression analyses showed that, compared with surgery group, patients in nonsurgery (RBR and NR) groups tend to be older, T4, N0/ $\mathrm{NX}$, triple-negative and visceral metastatic. For both all-cause survival and BCSS, non-surgery, advanced T stage, triple-negative $\mathrm{BC}$ (TNBC) and visceral metastases were significant risk factors. Primary tumour surgery showed benefits for both all-cause survival ( $\mathrm{HR}=0.44$, $95 \% \mathrm{Cl}=0.39-0.49, \mathrm{p}<0.0001)$ and $\mathrm{BCSS}(\mathrm{HR}=0.43,95 \%$ $\mathrm{Cl}=0.38-0.49, \mathrm{p}<0.0001)$. However, after propensity score matching, primary tumour surgery failed to demonstrate significant benefits for TNBC ( $\mathrm{HR}=0.96,95 \% \mathrm{Cl}=0.60$ $1.53, p=0.851)$ and patients with visceral metastases ( $\mathrm{HR}=0.90,95 \% \mathrm{Cl}=0.60-1.36, \mathrm{p}=0.62$ ).

Conclusion Surgery was associated with prolonged survival in stage IV breast cancers, but not in patients with TNBC and visceral metastases.

\section{INTRODUCTION}

Although the 5-year survival rate of patients with breast cancer (BC) has reached $90 \%$ in 2010s, BC is still the leading cause of tumour-related death among women. ${ }^{1}$ Specifically, metastatic BC (MBC) is a devastating cause of morbidity and mortality in women.

\section{Strengths and limitations of this study}

- Surveillance, Epidemiology, and End Results (SEER) database is a large database of US patients with cancer, but no chemotherapy information was included in this database.

- In SEER database, non-surgery patients were divided into recommended but refuse group and not recommended group, which subdivided patients more precisely.

- Propensity score matching analysis was performed to eliminate the effect of unbalanced variables between surgery group and surgery recommended but refused group.

- Stratified analysis was performed to evaluate the effect of primary surgery on patients with specific clinical features.

Clinically, MBCs are treated with systematic therapy, including local surgery, systematic chemotherapy, targeting therapy and immunotherapy. Although early study suggested that chemotherapy and targeting therapies with local management improve survival outcomes, $^{2} 3$ there is no standard local treatment strategy for MBC. Therefore, for patients with MBC who were first diagnosed as stage IV with an intact primary breast tumour, how to select the appropriate treatment of the primary tumour in patients with $\mathrm{BC}$ with brain metastasis remains a problem for oncologists.

This problem has been studied for years. Based on Surveillance, Epidemiology, and End Results Programme (SEER) registry data from 1988 to 2011, Thomas et al suggested that primary tumour surgery is associated with improved survival. ${ }^{4}$ Based on a single-centre data, Kim's group found that the removal of primary breast tumours was a significant independent beneficial predictor for survival. ${ }^{5}$ 
However, in the open-label randomised controlled trial, only patients with bone and visceral MBC were included. ${ }^{6}$ These work provided controversial findings which could be caused by patient heterogeneity. Therefore, it is important to study the potential subgroup patients who had favourable features and might be beneficial from locoregional treatment of the primary breast tumours. Even if the routine local therapy is not recommended for all patients with MBC, it is likely that the selected individuals could still benefit from it. ${ }^{7}$

In this study, in order to identify the subgroup patients who might benefit from primary tumour surgery, we collected 13618 patients diagnosed with MBC from January 2010 to December 2015 using SEER database. Among them, 4112 patients underwent primary BC surgery, 9020 patients were not recommended for surgery, and 486 patients were recommended for surgery but refused eventually.

\section{METHODS}

\section{Data collection}

To evaluate the treatment outcome of primary site surgery in MBC, we used the SEER*Stat database, which was released by the Surveillance Research Program at NCI in 2019. ${ }^{8}$ Patients diagnosed with MBC (site: breast (C50.0C50.9); AJCG stage 7th ed (2010+)='IV', 'IVNOS', 'IVA', 'IVA1', 'IVA2', 'IVB', 'IVC') between 2010 and 2015 were identified in the SEER 18 Regs Research Data+Hurricane Katrina Impacted Louisiana Cases, Nov 2018 Sub (19732016 varing) incidence database. Patients with a history of any type of cancer or non-BC-specific mortality were excluded from this study. Patients who received primary site surgery were compared with both patients who were recommended for surgery but refused, and patients who were not recommended for surgery and also did not receive surgery.

As shown in online supplemental figure S1, the exclusion criteria are: (1) patients with unknown bone or visceral metastatic information; (2) patients with unknown cancer-directed surgery information, or patients died before recommended surgery; (3) patients were not recommended for surgery due to other unknown condition; (4) patients with unknown death cause.

\section{Cohort description}

Based on the surgery status at primary site, the patients were categorised into three groups: patients with primary tumour surgery performed (SP), patients who were recommended for primary tumour surgery but not performed due to refusal (or other unknown reason) (RBR), and patients who were not recommended for primary tumour surgery and surgery was not performed. The primary outcome measure was all-cause mortality, and the secondary outcome measure was BC-specific mortality. The candidate risk factors for overall survival (OS) included cancer-directed surgery, age, year of diagnosis, race, $\mathrm{T}$ stage, $\mathrm{N}$ stage, human epidermal growth factor receptor 2 (HER2) status, oestrogen receptor (ER) and progesterone receptor (PR) status, and metastatic sites.

\section{Statistic methods}

Numeric variable age was summarised as the mean (SD) and median (IQR). Categorical variables were reported as counts (\%). An analysis of variance was used to compare age among three local treatment subgroups. Mentel-Haenszel $\chi^{2}$ tests or Fisher's exact tests $(n<5)$ were used to compare categorical variables between the three subgroups. The Cox proportional hazards regression was performed to estimate the HR to identify the risk factors for BC-specific mortality and all-cause mortality.

\section{Stratified Cox regression}

In order to search for patients who might benefit from primary tumour surgery, the stratified Cox proportional hazards regression analysis was performed to evaluate the effect of primary surgery on patients with specific clinical features. For example, based on patients' metastatic sites, patients with stage IV BC were categorised into levels: (1) lymph node mets-only, (2) bone mets-only, (3) visceral mets (one organ), (4) bone+visercal mets (one organ), visceral mets ( $\geq 2$ organs) with/without bone metastatic.

For patients who were recommended for primary tumour surgery, they were supposed to benefit from local primary tumour surgery. But a small group of them refused surgery or surgery was not performed due to unknown reasons. If this small group of patients received surgery, they might have a better prognosis. Thus, to evaluate the effect of surgery on survival and search for patients who might benefit from surgery, patients who were recommended for surgery but refused were compared with the patients who received surgery for each clinical feature by using stratified Cox model.

\section{Propensity score matching}

However, there were still unbalanced variables between primary surgery performed group and RBR group. Thus, a 1:1 propensity score matching (PSM) analysis was performed to reduce the potential bias between the two groups. Propensity scores were calculated through logistic regression for each patient in compared groups. The covariates in the logistic regression included diagnosis year, race, $\mathrm{T}$ stage, $\mathrm{N}$ stage, metastasis site, HER2/ HR status and radiation. Patients in the two groups were matched based on the propensity score. Covariate balance between two groups was examined by standard differences. Survival comparison was then performed for the matched patients using the same methods as those in the unmatched patients.

For both raw dataset and PSM dataset, the KaplanMeier method was used to plot the survival distributions, and the logrank test was used to assess the differences in survival experience between the subgroups. The stratified Cox regression was used to illustrate the treatment variance among subgroups. Because of the heterogeneity 
Table 1 Patient cohort $(n=13618)$

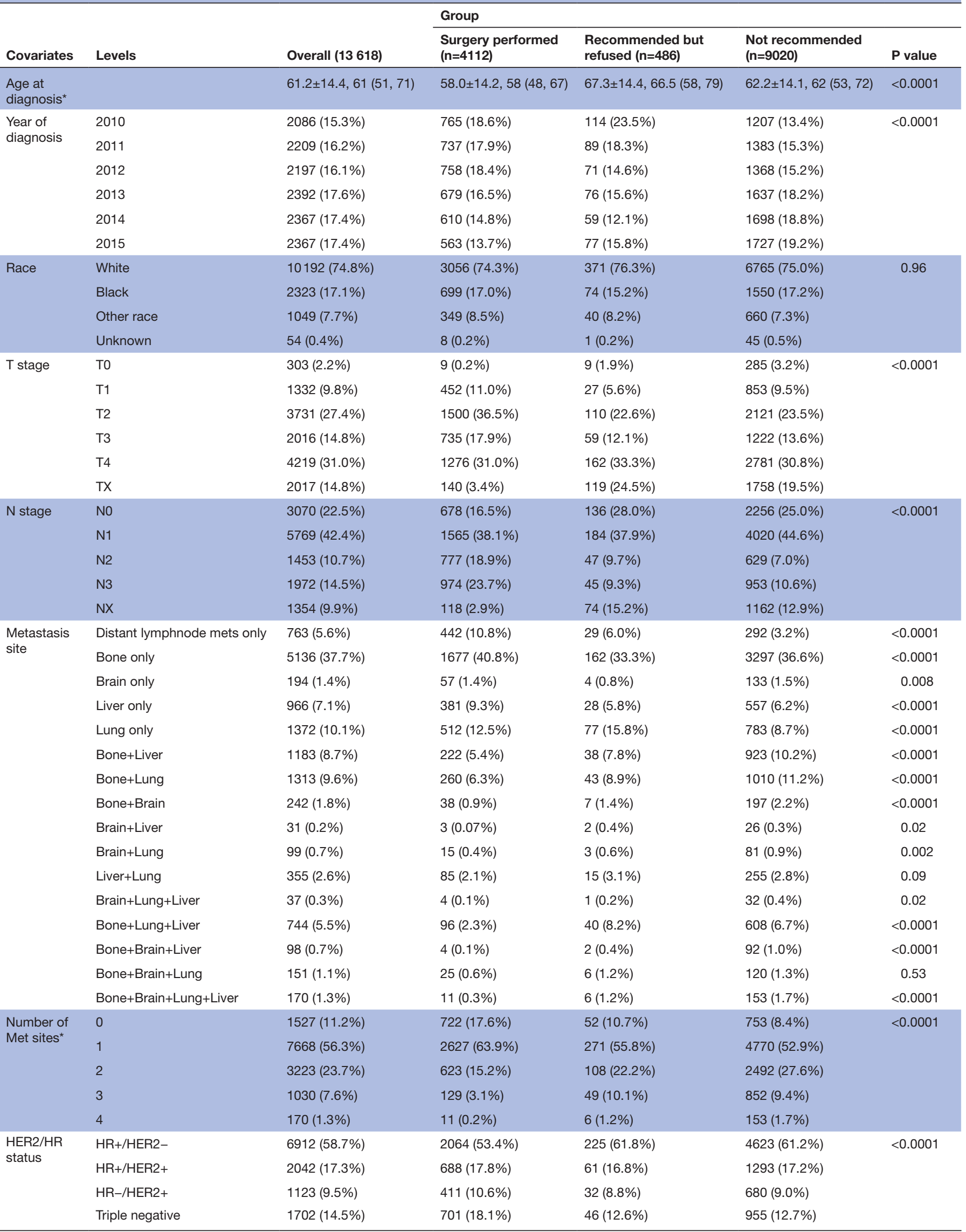


Table 1 Continued

\begin{tabular}{|c|c|c|c|c|c|c|}
\hline \multirow[b]{2}{*}{ Covariates } & \multirow[b]{2}{*}{ Levels } & \multirow[b]{2}{*}{ Overall (13 618) } & \multicolumn{4}{|l|}{ Group } \\
\hline & & & $\begin{array}{l}\text { Surgery performed } \\
(n=4112)\end{array}$ & $\begin{array}{l}\text { Recommended but } \\
\text { refused ( } n=486)\end{array}$ & $\begin{array}{l}\text { Not recommended } \\
(\mathrm{n}=9020)\end{array}$ & $P$ value \\
\hline \multirow[t]{3}{*}{ HER2 } & Negative & 8688 (70.9\%) & $2771(70.0 \%)$ & 272 (71.4\%) & $5643(71.3 \%)$ & 0.001 \\
\hline & Positive & $3190(26.0 \%)$ & 1101 (27.8\%) & $96(25.2 \%)$ & $1993(25.2 \%)$ & \\
\hline & Borderline & $376(3.1 \%)$ & $86(2.2 \%)$ & $13(3.4 \%)$ & 277 (3.5\%) & \\
\hline \multirow[t]{2}{*}{ ER } & Negative & $3154(25.0 \%)$ & $1211(30.2 \%)$ & $87(21.7 \%)$ & $1856(22.7 \%)$ & $<0.0001$ \\
\hline & Borderline & $11(0.09 \%)$ & $2(0.05 \%)$ & $2(0.5 \%)$ & 7 (0.1\%) & \\
\hline \multirow[t]{3}{*}{ PR } & Negative & 4978 (39.9\%) & $1758(43.9 \%)$ & $146(36.9 \%)$ & 3074 (38.1\%) & $<0.0001$ \\
\hline & Positive & 7471 (59.9\%) & 2245 (56.0\%) & 247 (63.3\%) & $4979(61.7 \%)$ & \\
\hline & Borderline & $28(0.2 \%)$ & $5(0.1 \%)$ & $3(0.6 \%)$ & $20(0.2 \%)$ & \\
\hline
\end{tabular}

*Age at diagnosis was continuous varible and was compared by using ANOVA analysis among three local treatment subgroups.

†Number of met sites represented the number of metastatic lesions which existed at bone, liver, lung, brain or distant lymphnodes. This variable did not include the number of metastatic lesions at other distant sites.

ER, oestrogen receptor; PR, progesterone receptor.

in patient characteristics, PSM was applied to adjust for baseline differences and reduce confounding. PSM was performed to compare patients with surgery performed and surgery recommended but patients refused. Propensity matching was assessed using balance diagnostics and standardised differences. All tests of hypotheses were twosided and conducted at 0.05 level of significance. Statistical analyses were conducted using SAS, V.9.4. Random forest tree plots were drawn by suing 'forestplot' package in R V.3.6.0.

\section{Patient and public involvement statement}

Patients and public were not involved in the study.

\section{RESULTS}

\section{Demographic and clinical characteristics}

Of 13618 patients with stage IV BC who were diagnosed from January 2010 to December 2015, 4112 patients underwent primary BC surgery, 9020 patients were not recommended for surgery and 486 patients were RBR surgery. Treatment characteristics across groups are shown in table 1. Patients with SP were younger (58.0 \pm 14.2 years old) than patients who are not recommended for surgery $(62.2 \pm 14.1$ years old) and patients who are RBR for surgery $(67.3 \pm 14.4$ years old $)$. There was no racial disparity and diagnosis year difference among treatment subgroups. The number of patients who received surgery gradually decreased from 765 in 2010 to 563 in 2015 , while the patients who were not recommended for surgery gradually increased from 1207 in 2010 to 1727 in 2015.

For these stage IV patients, both the primary tumour $(\mathrm{T})$ stage and the local lymphnode $(\mathrm{N})$ stage varied among three subgroups. The percentage of T0-T2 patients in SP subgroup $(47.7 \%(\mathrm{n}=1961))$ was higher than that in RBR subgroup $(30.1 \%(\mathrm{n}=146))$ and Surgery not recommended (NR) subgroup (36.2\% ( $\mathrm{n}=3259))$ patients. But the percentage of N0 patients in SP group (16.5\%) was lower than RBR and NR subgroups (28.0\% and 25.0\%, respectively). These data suggested that patients who received surgery were more likely have an intact primary tumour at early $\mathrm{T}$ stage, but would develop lymphnode metastases (table 1). As for the metastatic lesions, the proportions of patients with bone-only metastasis are $40.8 \%(\mathrm{n}=895)$ in SP subgroup, higher than those in RBR subgroup (33.3\%) and NR subgroup (36.6\%). Patients with visceral metastases with/without bone metastases (number of metastatic organs $\geq 2$ ) were more likely not to be recommended for primary tumour surgery. Among four HER2/HR status categories, the proportion of $\mathrm{HR}+$ / HER2- subtype was higher in RBR and NR groups $(61.8 \%$ and $61.2 \%$, respectively) than SP group (53.4\%). The proportion of triple-negative subtype was higher in SP group $(18.1 \%)$ than RBR and NR groups $(12.6 \%$ and $12.7 \%$, respectively).

\section{Risk factors for all-cause mortality and BC-specific mortality}

Univariate Cox regression analyses was performed to evaluate the risk factors for all-cause mortality and BC-specific mortality (ACMBCM). As shown in figure 1, the hazards of ACMBCM among patients who were RBR surgery were 2.28 times (95\% CI: 2.04-2.55) and 2.31 times (95\% CI: 2.06-2.60), respectively, higher than that among those with surgery performed. The hazards of ACMBCM among patients who were not recommended for surgery were 1.91 times (95\% CI: $1.81-2.00)$ and 1.92 times (95\% CI: 1.82-2.02), respectively, higher than that for patients with surgery performed. Based on these results, surgery was associated with improved survival in the unmatched cohorts.

Risk factors, including old age, black race and advanced $\mathrm{T}$ stage were significant for both all-cause mortality and BC-specific mortality. TNBC was also a significant risk factor. Besides, the visceral metastases (with/without bone metastases) was also related to a worse OS. In addition, compared with no bone or visceral metastases, patients 


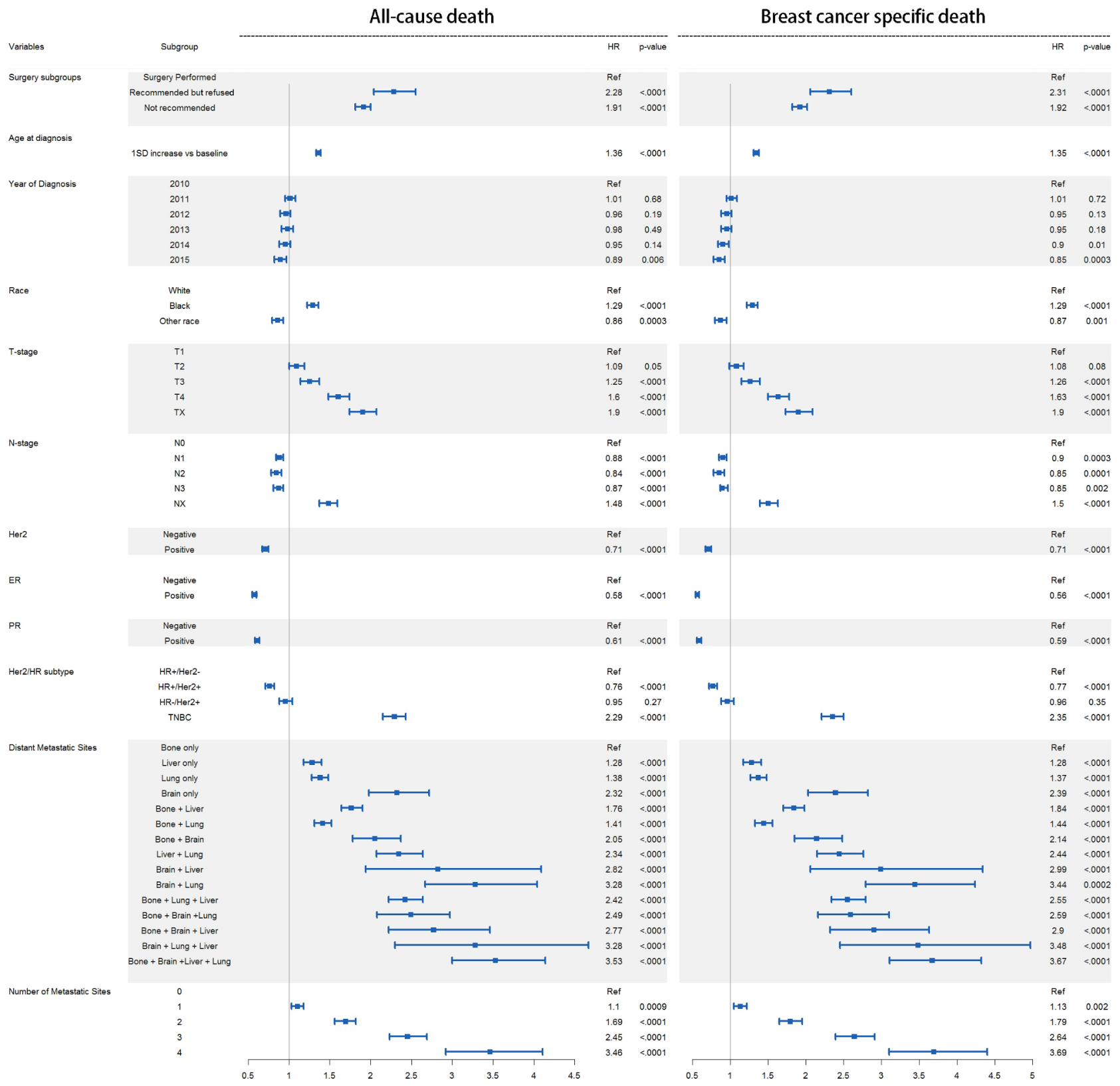

Figure $1 \mathrm{HRs}$ (with 95\% Cl) of clinical candidate variables for all-cause mortality (left panel) and breast cancer-specific mortality (right panel).

with bone or visceral metastases (in one to four organs) had significant poor prognosis. ER-positive, PR-positive and HER2-positive were all protective factors against both all-cause mortality and BC-specific mortality.

\section{Effect of primary tumour surgery stratified by candidate variables}

To evaluate the effect of surgery on survival and search for patients who might benefit from surgery, patients who were recommended for surgery but refused were compared with the patients who received surgery in subgroups, stratified by the significant candidate variables discussed previously. As shown in figure 2, in unmatched raw dataset (blue), compared with patients who were RBR surgery, primary tumour surgery was beneficial to both all-cause survival and BCSS in all stratified subgroups (blue line).

However, the distribution of patients between SP and RBR group was unbalanced. Therefore, 1:1 PSM was performed. After PSM, all candidate variables, including age at diagnosis, year of diagnosis, race, $\mathrm{T}$ stage, $\mathrm{N}$ stage, distant metastatic sites, and HR/HER2 subtypes, were balanced between surgery-performed group and surgery-RBR group (online supplemental table S1). In PSM dataset, primary tumour surgery still show significant beneficial effect for all-cause survival in most stratified subgroups (figure 2, yellow line). But for patients with visceral metastases, surgery showed minimal benefit. In addition, for patients with TNBC, surgery also did not improve BCSS. 


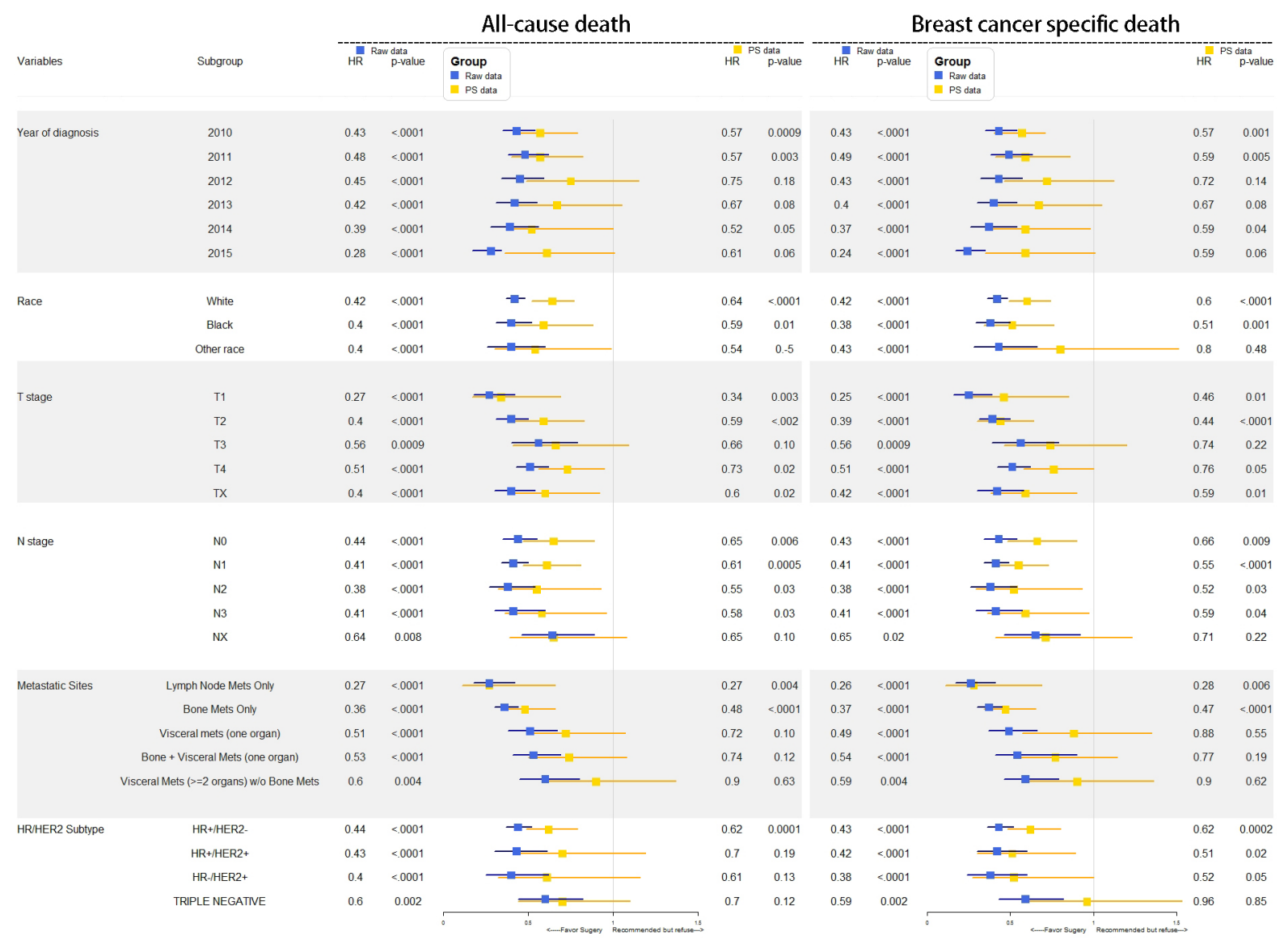

Figure 2 HRs (with 95\% Cl) of primary tumour surgery versus surgery recommended but not performed for all-cause mortality (left panel) and breast cancer-specific mortality (right panel) in patients with specific clinical features (stratified Cox regression analyses) in raw dataset (blue line) and propensity-score matched (PSM) dataset (yellow line).

\section{Kaplan-Meier curves and survival analyses}

Survival analysis showed that, before PSM, the median all-cause survival and BCSS for patients who were recommended for surgery but refused were both 16 months. After PSM, the medians were 18 and 19 months, respectively. The median all-cause survival and BCSS for patients who received primary tumour surgery were 44 and 46 months, respectively (Logrank $\mathrm{p}<0.0001$, figure $3 \mathrm{left}$ panel). After PSM, the medians were 31 and 33 months, respectively (Logrank $\mathrm{p}<0.0001$, figure 3 right panel). PSM results seemed to confirm the benefit of primary surgery on patients with stage IV BC.

However, in the subgroup of patients with visceral metastases, the benefit of primary surgery on all-cause survival and BCSS was not significant. Figure 4 (left panel) demonstrated that in raw dataset, the median all-cause OS for surgery-performed patients with bone-only metastases, one organ visceral metastases, one organ visceral metastases plus bone metastases, and two organs' visceral metastases with or without bone metastases were 53, 34, 34, and 18 months, respectively. For all-cause OS for surgery RBR patients, the medians were $20,15,15$, and 8 months, respectively. Surgery significantly improved allcause OS and BCSS (online supplemental figure S2, left panel) in raw dataset. However, in PSM dataset, surgery improved neither the all-cause OS (figure 4, right panel) nor BCSS (online supplemental figure S2, right panel) in patients with one organ visceral metastases, one organ visceral metastases plus bone metastases, and two organs' visceral metastases with or without bone metastases. The median all-cause OS for surgery-performed patients with one organ visceral metastases, one organ visceral metastases plus bone metastases, and two organs' visceral metastases with or without bone metastases were 20, 25, and 15 months, respectively. For surgery RBR patients, the medians were $20,15,15$, and 8 months, respectively

\section{DISCUSSION}

In this study, we collected 13618 patients with stage IV BC who were diagnosed between 2010 and 2015 from SEER database. We confirmed that primary tumour surgery improved both all-cause OS and BCSS. However, we found that patients who received surgery were younger, and tended to be in T1/T2 stage and have bone-only metastases. Therefore, we applied PSM method to address for the differences. After PSM, we found that compared with patients who were recommended for surgery but refused, patients who received surgery did not survival longer if they had visceral metastases (liver, lung or brain metastases, figures 2 and 4). Patients with bone-only metastases 

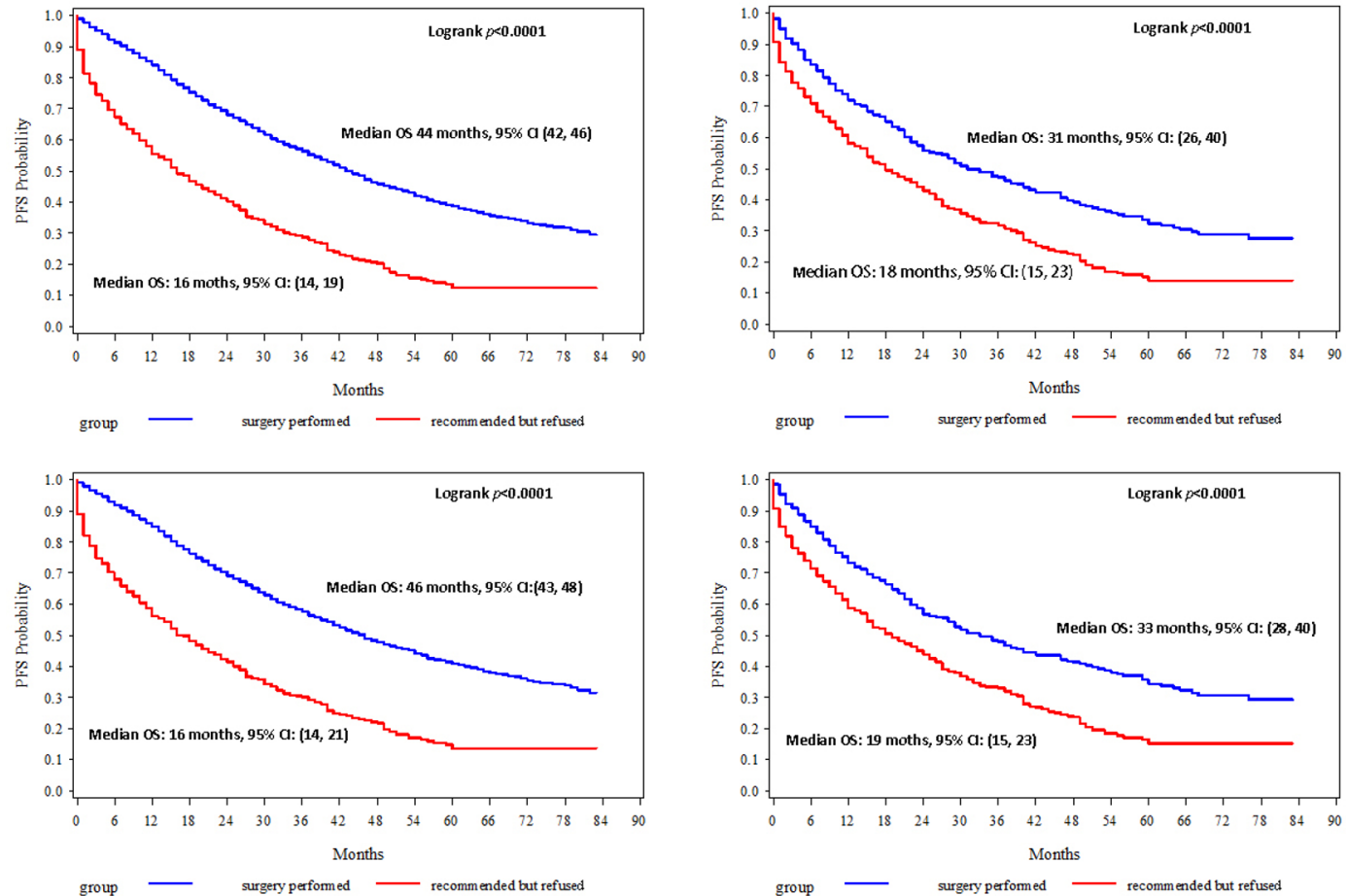

Figure 3 Kaplan-Meier curves of all-cause survival (upper) and breast cancer-specific survival (lower) in raw dataset (left panel) and propensity-score matched (PSM) dataset (right panel).

still had significant OS benefit from primary tumour surgery after PSM.

Oligometastatic tumours are characterised by solitary or few metastatic lesions that are usually limited to a single organ. ${ }^{9}$ Patients with oligometastatic BC often have controllable symptoms, and larger chance to cure. Technical improvements in surgery and radiotherapy have introduced the option of metastasis-directed ablative therapies as an adjunct or alternative to standardof-care systemic therapies. ${ }^{10}$ In this study, we found that most patients with MBC have benefited from locolregional primary tumour surgery (figure 2). In propensity score (PS)-matched dataset, only patients with bonelimited MBC have significantly benefited from locolregional primary tumour surgery (figure 2 ). The HR values suggested a protective effect of surgery, especially for allcause OS. But $p$ values were not significant due to the relatively small sample size after PSM.

Partial or total mastectomy with radiotherapy (RT) performed best in prolonging BCSS in patients with MBC with different HR/HER2 subtype. TNBC is considered as the most aggressive subtype among all four HR/HER2 subtypes, with rapid metastatic rate. ${ }^{11-13}$ Due to the lack of effective therapy after metastasis, TNBC is associated with a poor prognosis. ${ }^{14}$ Currently, multiple targeting therapy strategies are under clinical research or preclinical research, including poly ADP-ribose polymerase (PARP) inhibitor, ${ }^{15}$ vascular endothelial growth factor receptor (VEGR) inhibitor, ${ }^{16}$ fibroblast growth factor receptor (FGFR) inhibitor ${ }^{17}$ and so on. Here, we found that primary tumour surgery did not benefit patients with TNBC in prolonging BCSS in PSM dataset. But for all-cause OS, the HR value of 0.70 still indicated a protective effect of surgery.

SEER database is a unique public resource, allowing the examination of patient characters and experiences of treatments among a large US population of individuals diagnosed with malignancies. This study serves as a complementary part to the previous studies ${ }^{18} 19$ by using SEER dataset. Since SEER dataset only include HR/ HER2 subtype information from 2010, this study used SEER data from 2010 to 2015. Localregional treatment techniques, including the surgical and RT techniques, have improved significantly after 2000 . Thus the survival rate of BC increased from $70 \%$ in 1970 s to $90 \%$ in 2000 s. The prognosis of patients diagnosed in 2010 cannot be compared with the prognosis of patients diagnosed in 1980. Therefore, in this study, we only included patients who diagnosed from 2010 to 2015. Such a selection might decrease the number of patients in study, but this group of patients is more representative to the current treatment experience and techniques.

This study still has several limitations. First, since the RBR group had relatively small sample size $(n=486)$, after 1:1 PSM, only about $12 \%$ of patients in surgery group remained. Therefore, propensity-score matched dataset might miss some features of surgery patients. Second, due to the missing information of radiotherapy in SEER database, the effect of radiation therapy was not considered in this study. Third, SEER dataset does not have the chemotherapy information. But patients in SEER database who were registered in hospital should have received standardised chemotherapy according to clinical guidelines. So, in this study, we suppose that all patients 

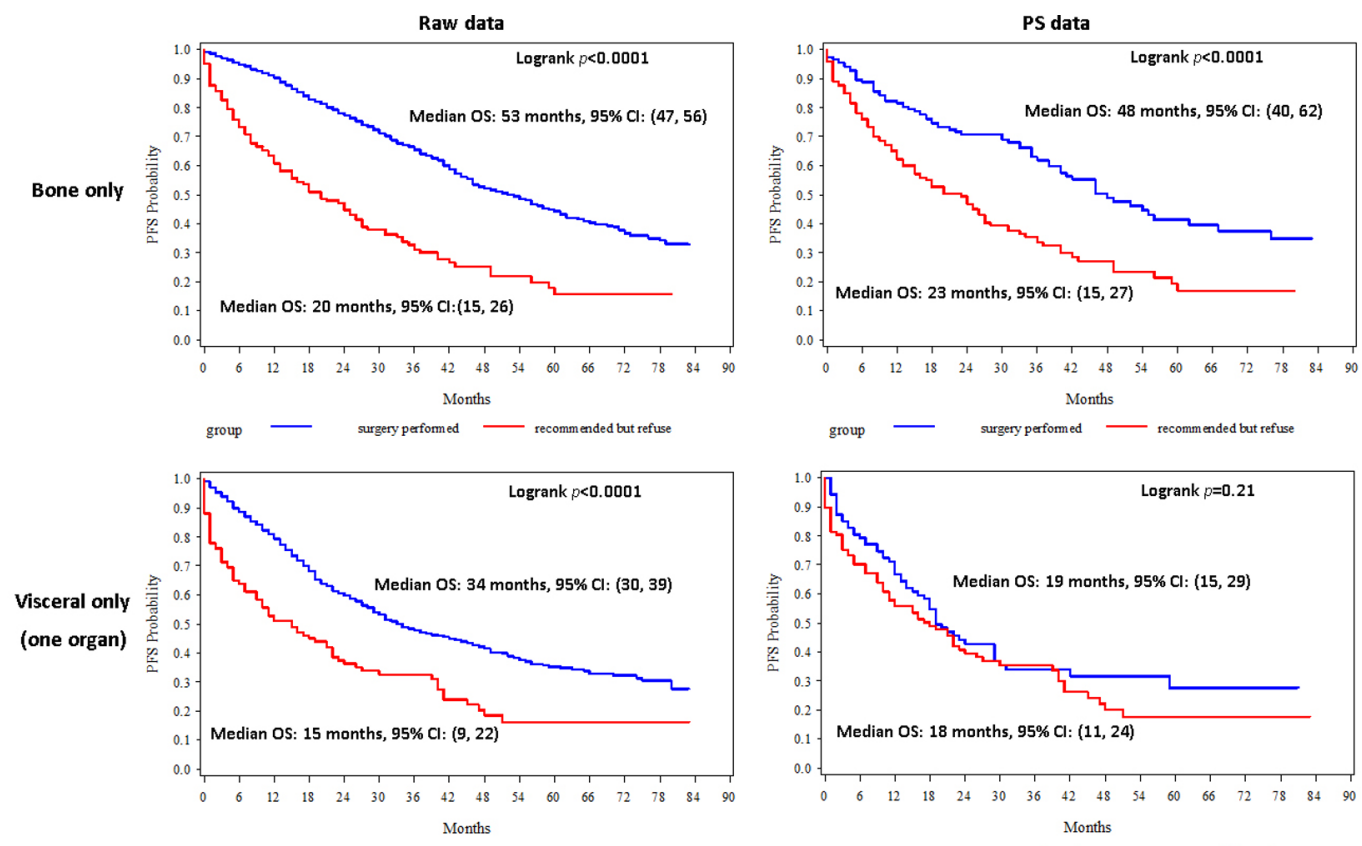

group - surgery performed - recommended but refus

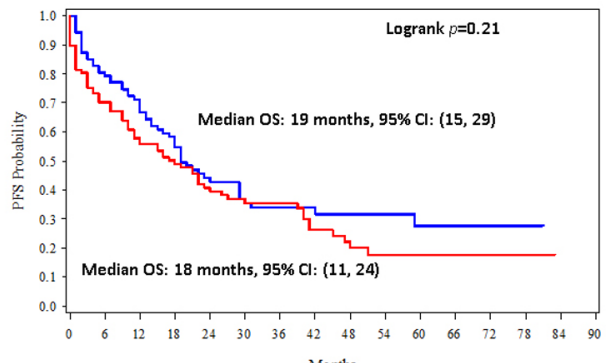

group - $\quad$ surgery performed

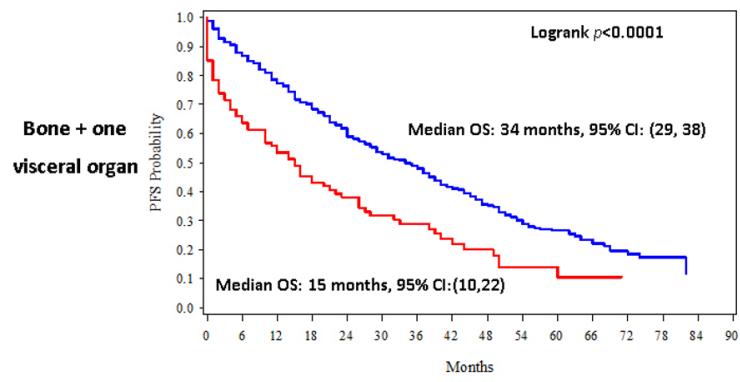

group - $\quad$ surgery pefformed $\quad$ recommended but refuse
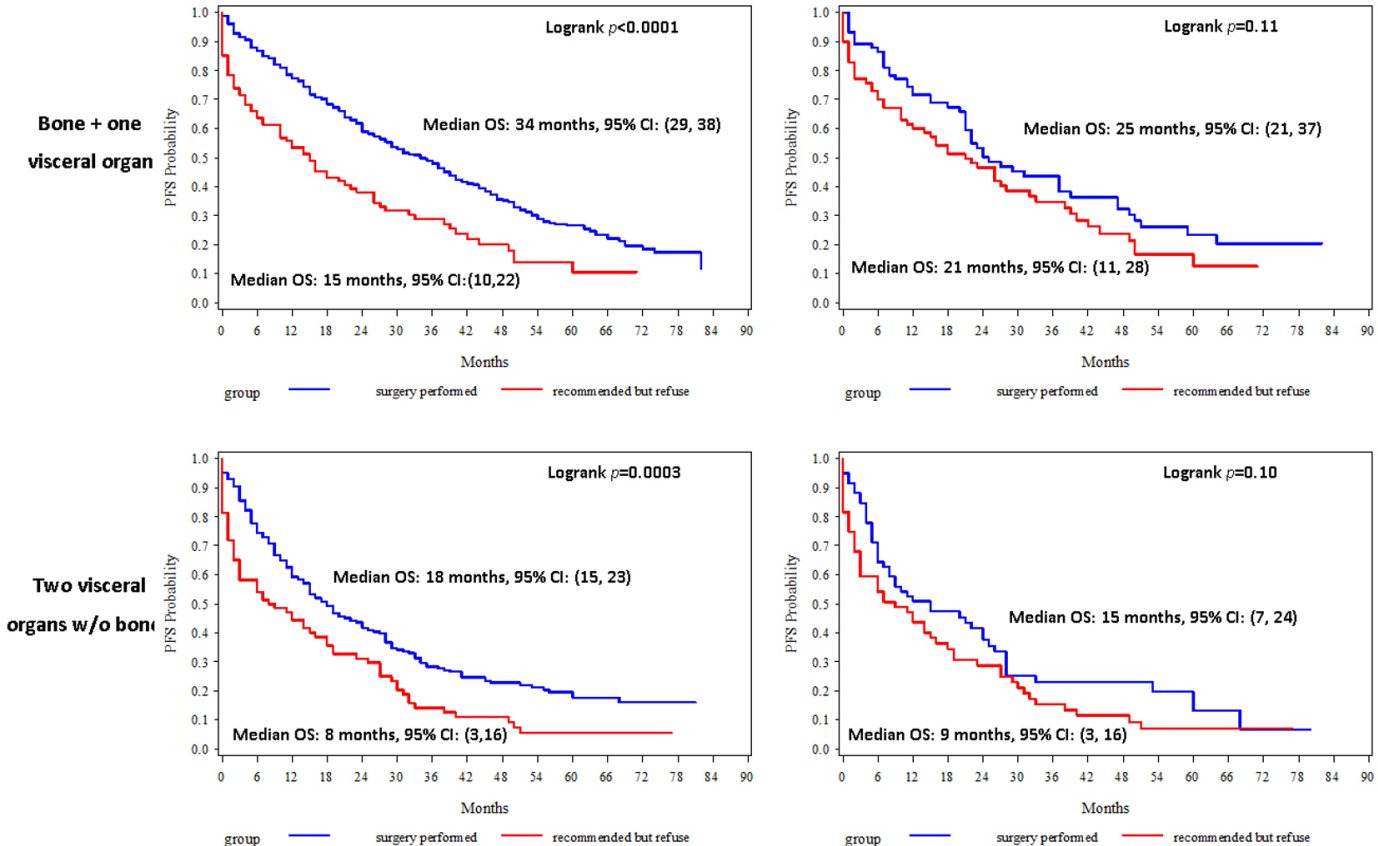

Figure $4 \mathrm{KM}$ curves of all-cause survival in four subgroups of patients (bone-only metastases, visceral-only (one organ) metastases, visceral (one organ) plus bone metastases and visceral (two organs) with or without bone metastases) in raw dataset (left panel) and propensity-score matched (PSM) dataset (right panel).

received standard treatment, including chemotherapy. In this study, we use propensity score match to balance the important clinical variables that are available in SEER database, including age, $\mathrm{T}$ stage, $\mathrm{N}$ stage, metastatic site and so on. These variables are also influential variables for chemotherapy. Even there is no chemotherapy information in SEER database, the variables in SEER database could still reflect most clinical features of patients.

Further large-populational clinical observational study would be needed to comprehensively investigate the effect of surgery on patients with stage IV BC. In conclusion, not all patients with stage IV primary BC would benefit from primary tumour resection. Instead, subgroup of patients with specific clinical features (bone-only metastases, small primary tumour size, HR-positive, HER2-positive and so on) would benefit from primary tumour resection.

\section{Author affiliations}

${ }^{1}$ Hunan Cancer Hospital, Central South University, Changsha, China

${ }^{2}$ Department of Breast Cancer Surgical Oncology, Hunan Cancer Hospital,

Changsha, China

${ }^{3}$ Department of Neurology, Hunan Provincial People's Hospital, Changsha, China ${ }^{4}$ ICF, Atlanta, Georgia, USA

${ }^{5}$ Xiangya Hospital of Central South University, Changsha, China 
${ }^{6}$ Clinical Research Center for Breast Cancer Control and Prevention in Hunan Province, Changsha, China

${ }^{7}$ College of Information and Intelligence, Hunan Agricultural University, Changsha, China

Acknowledgements This study used the SEER 18 Regs research database as the data source. The interpretation and reporting of these data are the sole responsibility of the authors. The authors acknowledge the efforts of the National Cancer Institute; the SEER Programme tumour registry and the Information Management Service for the creation and distribution of the SEER ${ }^{\star}$ Stat database.

Contributors Wei Peng, Yu Tang, Xiaoho Hu and Ying He collected the data and prepared the manuscript. Quchang Ouyang, Ning Xie and Can Tian provide the medical resources. Wei Peng, Chongyu Hu and Xiangyan Liu designed the study and interpreted the patient data regarding surgery and survival. Haoyu Zhou, Xiao Wang and Zhe-Yu Hu performed the data analysis. All authors read and approved the final manuscript.

Funding This work was supported by Hunan Provincial Science and Technology Department Project grant number 2018SSK2120, 2018SK2124, 2019SK2032 and 2019JJ50360, Health and Family Planning Commission of Hunan Province Project grant number C2019070 and B2019089, Changsha City Technology Program grant numbers kq1901076 and kq2004125, and National Science Foundation of China 61972147.

Competing interests None declared.

Patient consent for publication Not applicable.

Ethics approval This study used the SEER 18 Regs research database as the data source. The SEER database is approved by NIH Ethics Programme (both the NIH Ethics Office and individual ethics programme in each Institute and Centre).

Provenance and peer review Not commissioned; externally peer reviewed.

Data availability statement Data are available upon reasonable request. Based on SEER website https://www.cancer.gov/policies/accessibility, the National Cancer Institute ( $\mathrm{NCl}$ ) provides access to all individuals seeking information on http://www. cancer.gov, including individuals who are disabled. To provide this information, the $\mathrm{NCl}$ website complies with Section 508 of the Rehabilitation Act (as amended). This study used SEER ${ }^{\star}$ Stat database released in the SEER 18Regs Research Data + Hurricane Katrina Impacted Louisiana Cases, Nov 2016 Sub (1973-2013 varying) incidence database. Consent for publication The National Cancer Institute (NCl) SEER database is free for public use. On SEER website: https://www.cancer.gov/ policies/foia, we are informed that $\mathrm{NCl}$ has a wealth of information available in both published and electronic formats. On the website https://www.cancer.gov/policies/ copyright-reuse, we are informed that most of the information on $\mathrm{NCl}$ website (https://www.cancer.gov/) is in the public domain and is not subject to copyright restrictions. No special permission is required to use or reproduce public domain material. Extra data are available by emailing Wei Peng pengwei19810404@163. com.

Supplemental material This content has been supplied by the author(s). It has not been vetted by BMJ Publishing Group Limited (BMJ) and may not have been peer-reviewed. Any opinions or recommendations discussed are solely those of the author(s) and are not endorsed by BMJ. BMJ disclaims all liability and responsibility arising from any reliance placed on the content. Where the content includes any translated material, BMJ does not warrant the accuracy and reliability of the translations (including but not limited to local regulations, clinical guidelines, terminology, drug names and drug dosages), and is not responsible for any error and/or omissions arising from translation and adaptation or otherwise.

Open access This is an open access article distributed in accordance with the Creative Commons Attribution Non Commercial (CC BY-NC 4.0) license, which permits others to distribute, remix, adapt, build upon this work non-commercially, and license their derivative works on different terms, provided the original work is properly cited, appropriate credit is given, any changes made indicated, and the use is non-commercial. See: http://creativecommons.org/licenses/by-nc/4.0/.

\section{ORCID iDs}

Yu Tang http://orcid.org/0000-0002-9532-4459

Zhe-Yu Hu http://orcid.org/0000-0003-0932-9745

Wei Peng http://orcid.org/0000-0002-4166-2014

\section{REFERENCES}

1 DeSantis C, Ma J, Bryan L, et al. Breast cancer statistics, 2013. CA Cancer J Clin 2014;64:52-62.

2 Wang-Gillam A, Li C-P, Bodoky G, et al. Nanoliposomal irinotecan with fluorouracil and folinic acid in metastatic pancreatic cancer after previous gemcitabine-based therapy (NAPOLI-1): a global, randomised, open-label, phase 3 trial. Lancet 2016;387:545-57.

3 Zhang Q, Chen J, Yu X, et al. Systemic treatment after whole-brain radiotherapy may improve survival in RPA class II/III breast cancer patients with brain metastasis. J Neurooncol 2013;114:181-9.

4 Thomas A, Khan SA, Chrischilles EA, et al. Initial surgery and survival in stage IV breast cancer in the United States, 1988-2011. JAMA Surg 2016;151:424-31.

$5 \mathrm{Kim} \mathrm{HJ}$, Kang E, Kim JH, et al. Survival benefit of surgical removal of primary tumor in patients with stage IV breast cancer. Clin Breast Cancer 2018;18:e1037-44.

6 Badwe R, Hawaldar R, Nair N, et al. Locoregional treatment versus no treatment of the primary tumour in metastatic breast cancer: an open-label randomised controlled trial. Lancet Oncol 2015;16:1380-8.

7 Patrick J, Khan SA. Surgical management of de novo stage IV breast cancer. J Natl Compr Canc Netw 2015;13:487-93.

8 Xie Z-M, Sun J, Hu Z-Y, et al. Survival outcomes of patients with lobular carcinoma in situ who underwent bilateral mastectomy or partial mastectomy. Eur J Cancer 2017;82:6-15.

9 Pagani O, Senkus E, Wood W, et al. International guidelines for management of metastatic breast cancer: can metastatic breast cancer be cured? J Natl Cancer Inst 2010;102:456-63.

10 Westphal T, Gampenrieder SP, Rinnerthaler G, et al. Cure in metastatic breast cancer. Memo 2018;11:172-9.

11 He Z-Y, Wu S-G, Peng F, et al. Up-Regulation of Rfc3 promotes triple negative breast cancer metastasis and is associated with poor prognosis via EMT. Transl Oncol 2017;10:1-9.

12 Allaoui R, Bergenfelz C, Mohlin S, et al. Cancer-associated fibroblastsecreted CXCL16 attracts monocytes to promote stroma activation in triple-negative breast cancers. Nat Commun 2016;7:13050.

13 Das SG, Romagnoli M, Mineva ND, et al. miR-720 is a downstream target of an ADAM8-induced ERK signaling cascade that promotes the migratory and invasive phenotype of triple-negative breast cancer cells. Breast Cancer Res 2016;18:40.

14 Wein L, Loi S. Mechanisms of resistance of chemotherapy in earlystage triple negative breast cancer (TNBC). Breast 2017;34 Suppl 1:S27-30.

15 Santoni M, Romagnoli E, Saladino T, et al. Triple negative breast cancer: key role of tumor-associated macrophages in regulating the activity of anti-PD-1/PD-L1 agents. Biochim Biophys Acta Rev Cancer 2018;1869:78-84.

16 Diéras V, Campone M, Yardley DA, et al. Randomized, phase II, placebo-controlled trial of onartuzumab and/or bevacizumab in combination with weekly paclitaxel in patients with metastatic triplenegative breast cancer. Ann Oncol 2015;26:1904-10.

17 Sharpe R, Pearson A, Herrera-Abreu MT, et al. FGFR signaling promotes the growth of triple-negative and basal-like breast cancer cell lines both in vitro and in vivo. Clin Cancer Res 2011;17:5275-86.

18 Lizarraga I, Schroeder MC, Weigel RJ, et al. Surgical management of breast cancer in 2010-2011 seer registries by hormone and HER2 receptor status. Ann Surg Oncol 2015;22 Suppl 3:566-72.

19 Warschkow R, Güller U, Tarantino I. Improved survival after primary tumor surgery in metastatic breast cancer: a Propensity-adjusted, population-based seer trend analysis. Ann Surg 2016;263:1188-98. 